\title{
Six tips for successful virtual anesthesiology interviews in the COVID-19 era and beyond
}

\author{
Jody C. Leng, MD, MS • Edward R. Mariano, MD, MAS · Kariem El-Boghdadly, MBBS, \\ BSc (Hons), FRCA, EDRA, MSc
}

Received: 10 March 2021/Revised: 15 March 2021/Accepted: 16 March 2021/Published online: 24 March 2021

(C) This is a U.S. government work and not under copyright protection in the U.S.; foreign copyright protection may apply 2021

To the Editor,

In the spring of 2020, with the COVID-19 pandemic spreading rapidly, shelter in place orders and travel restrictions forced a dramatic transformation in the way we live and work. For academic medical centres, the process of interviewing and selecting applicants for anesthesiology residencies and fellowships had to go on.

For our regional anesthesiology fellowship, we were forced to conduct all our March 2020 interviews virtually for the first time since our interview dates had already been scheduled the prior year. Through the heroic efforts of our fellowship administrators and faculty volunteers, we hosted successful virtual interviews and filled each of our positions.

As a result of this first-hand experience, we learned valuable lessons that may be helpful for academic anesthesiology residency and fellowship programs and applicants throughout the rest of the COVID-19 pandemic and beyond. We present six tips (Figure) to make the virtual interview day successful for both the program and applicants: ${ }^{1-3}$

J. C. Leng, MD, MS · E. R. Mariano, MD, MAS ( $₫)$ Department of Anesthesiology, Perioperative and Pain Medicine, Stanford University School of Medicine, Stanford, CA, USA

e-mail: emariano@stanford.edu

Anesthesiology and Perioperative Care Service, Veterans Affairs Palo Alto Health Care System, Palo Alto, CA, USA

K. El-Boghdadly, MBBS, BSc (Hons), FRCA, EDRA, MSc Department of Anaesthesia and Perioperative Medicine, Guy's and St Thomas' NHS Foundation, London, UK

King's College London, London, UK

\section{Go virtual for all interviews}

To ensure the fairest process possible, we recommend hosting all interviews virtually. This prevents in-person interviewees from having an advantage over virtual interviewees and represents the most equitable solution since not all interviewees will have the financial means to travel to every program for interviews.

\section{Showcase your program}

Since interviewees will not be able to take a physical walking tour of the facilities or participate in informal conversations with faculty and trainees, we recommend investing in a high-quality video to give applicants a sense of the institutional culture. Such a video can be featured on the departmental website and social media platforms.

\section{Train your interviewers}

Interviewers, especially those who have not previously conducted virtual interviews, will need coaching on the videoconferencing platform, and we strongly recommend unconscious bias training as well.

\section{Do a test run}

Both interviewers and interviewees need to test their systems and surroundings before the actual interview day: lighting, background, microphone, camera, ambient noise, internet speed.

\section{Plan for glitches}

Something always goes wrong. We recommend having an administrator or program coordinator communicate with interviewers and interviewees via text messaging prior to the interview day so all parties have a direct contact in case of technical issues.

\section{Act like you would in person}

Although the interviews are virtual, it does not have to feel like it. Design a daily schedule that includes sufficient time for interviewing (e.g., $30 \mathrm{~min}$ ) and breaks in between (e.g., five minutes). One suggestion is to have each 


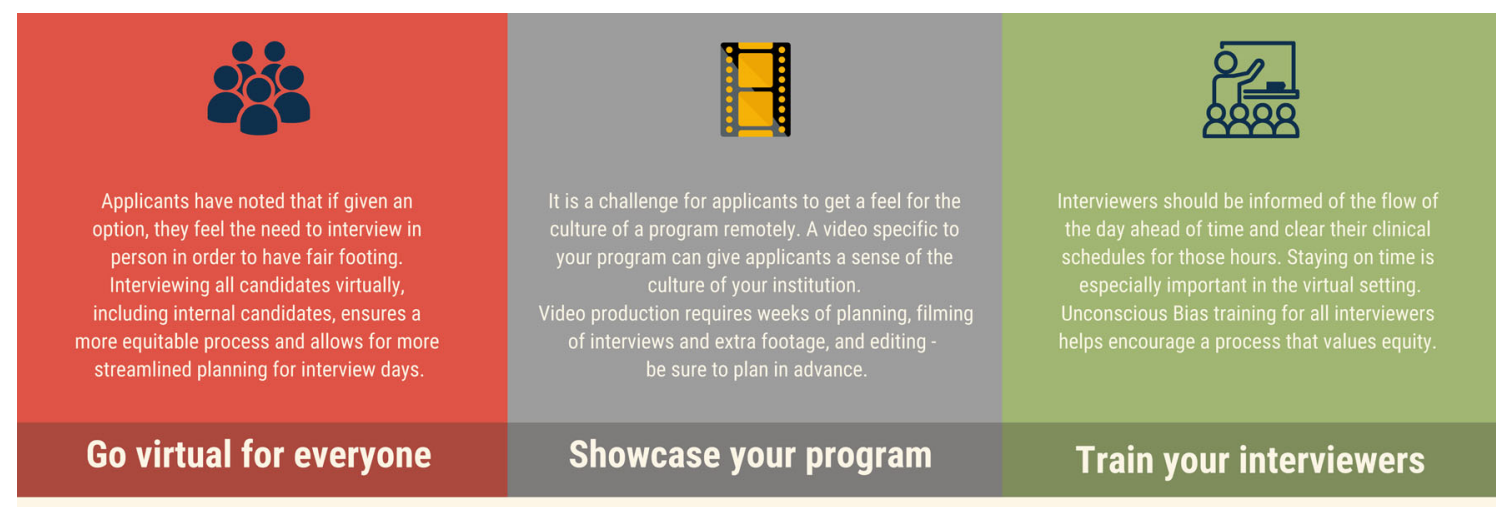

\section{Tips for Virtual Anesthesiology Interviews}

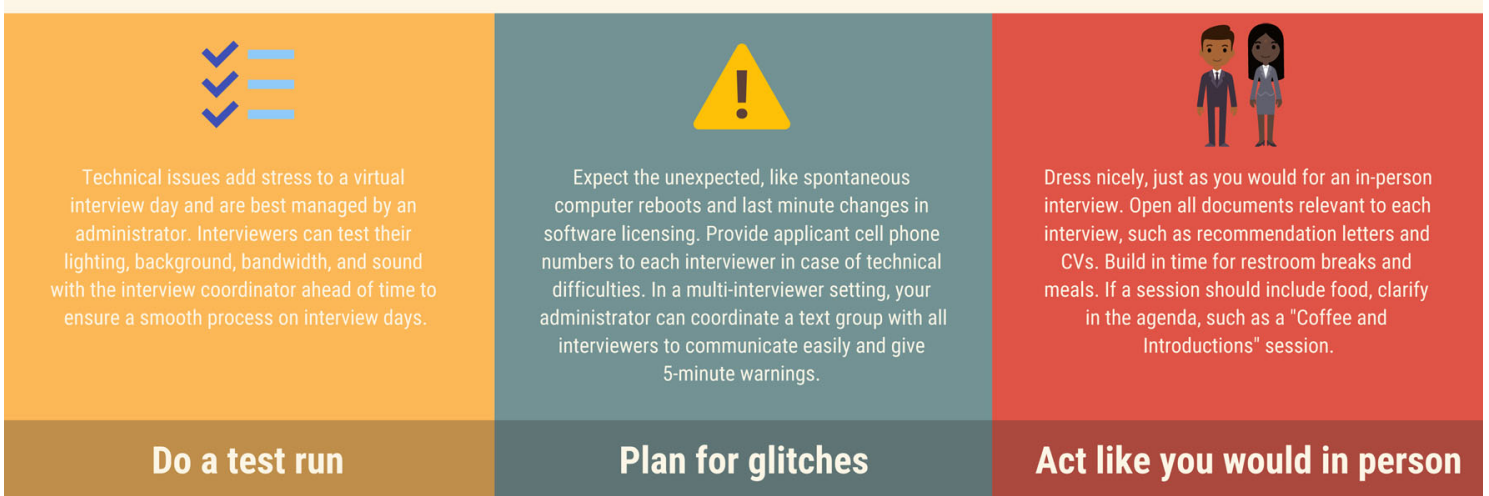

Figure Infographic highlighting six key suggestions for successful virtual anesthesiology interviews.

applicant assigned a unique virtual "room"; the interviewers $\log$ in and out, but the applicants stay logged in for the day. This helps keep the interviewers on time since a new interviewer logging in is a good reminder to wrap up, and the interviewees can easily take breaks by turning off their cameras and microphones without logging out.

Our summary infographic (Figure) can be shared with all stakeholders in the anesthesiology interview process: program directors, program coordinators and educators, interviewers, and interviewees. Programs may want to consider providing a similar infographic to applicants, perhaps posting on the program's website and social media platforms, to inform them both of what the program is doing to prepare for virtual interviews as well as what they can do as candidates. One example that was adapted for our regional anesthesiology fellowship program is posted on the program director's personal blog (https://www. edmariano.com/archives/2105).

While planning for virtual interviews for the foreseeable future may seem daunting, applying these important lessons should ensure an effective, efficient, and enjoyable interview experience for all involved.

Acknowledgements The authors are grateful to Bernadett Mahanay, Fellowship Program Supervisor, and Ashley Johnson, Fellowship Coordinator, in the Stanford Department of Anesthesiology, Perioperative and Pain Medicine. This work was supported with resources and the use of facilities at the Veterans Affairs Palo Alto Health Care System (Palo Alto, CA, USA). The contents do not represent the views of the Department of Veterans Affairs or the United States Government.

Disclosures and funding statement Kariem El-Boghdadly has received funding, research, or educational support from Ambu, GE Healthcare and Fisher and Paykel Healthcare. Jody C. Leng and Edward R. Mariano have no conflicts of interest to declare.

Editorial responsibility This submission was handled by Dr. Stephan K.W. Schwarz, Editor-in-Chief, Canadian Journal of Anesthesia/Journal canadien d'anesthésie. 


\section{References}

1. Davis MG, Haas MR, Gottlieb M, House JB, Huang RD, Hopson $L R$. Zooming in versus flying out: virtual residency interviews in the era of COVID-19. AEM Educ Train 2020; 4: 443-6.

2. Joshi A, Bloom DA, Spencer A, Gaetke-Udager K, Cohan RH. Video interviewing: a review and recommendations for implementation in the era of COVID-19 and beyond. Acad Radiol 2020; 27: 1316-22.
3. Deitte LA, Mian AZ, Esfahani SA, Hu JY. Going virtual: redesigning the interview experience. J Am Coll Radiol 2021; 18: 337-9.

Publisher's Note Springer Nature remains neutral with regard to jurisdictional claims in published maps and institutional affiliations. 